OPEN ACCESS

Edited by:

Chiang-shan R. Li, Yale University, USA

Reviewed by: Xin Di,

New Jersey Institute of Technology,

South China Normal University, China

${ }^{*}$ Correspondence: Judy Luigjes judyluigjes@gmail.com

Received: 11 December 2015 Accepted: 25 May 2016

Published: 14 June 2016

Citation:

Luigjes J, Figee M, Tobler PN, van den Brink $W$, de Kwaasteniet $B$, van Wingen $G$ and Denys D (2016) Doubt in the Insula: Risk Processing in Obsessive-Compulsive Disorder.

Front. Hum. Neurosci. 10:283. doi: 10.3389/fnhum.2016.00283

\section{Doubt in the Insula: Risk Processing in Obsessive-Compulsive Disorder}

\author{
Judy Luigjes ${ }^{1,2 *}$, Martijn Figee ${ }^{1,2}$, Philippe N. Tobler ${ }^{3}$, Wim van den Brink', \\ Bart de Kwaasteniet ${ }^{1}$, Guido van Wingen ${ }^{1,2}$ and Damiaan Denys ${ }^{1,4}$
}

${ }^{1}$ Department of Psychiatry, Academic Medical Center, University of Amsterdam, Amsterdam, Netherlands, ${ }^{2}$ Brain Imaging Center, Academic Medical Center, University of Amsterdam, Amsterdam, Netherlands, ${ }^{3}$ Laboratory for Social and Neural Systems Research, Department of Economics, University of Zurich, Zurich, Switzerland, ${ }^{4}$ Netherlands Institute for Neuroscience, The Royal Netherlands Academy of Arts and Sciences, Amsterdam, Netherlands

Extensive cleaning or checking of patients with obsessive-compulsive disorder (OCD) are often interpreted as strategies to avoid harm and as an expression of the widespread belief that OCD patients are more risk-averse. However, despite its clinical significance, the neural basis of risk attitude in OCD is unknown. Here, we investigated neural activity during risk processing using functional magnetic resonance imaging and simultaneously assessed risk attitude using a separate behavioral paradigm in OCD patients with different symptoms versus healthy controls (HCs). We found opposite insula responses to high versus low risk in OCD patients compared to HCs: a positive correlation between insula activity and risk-aversion in patients versus a negative correlation in controls. Although OCD patients overall were not more risk-averse than controls, there were differences between subgroups of OCD patients: patients with doubt/checking symptoms were more risk-averse than other patients. Taken together, OCD patients show a reversed pattern of risk processing by the insula compared to HCs. Moreover, the data suggest that increased activation of the insula signals an abnormal urge to avoid risks in the subpopulation of OCD patients with doubt and checking symptoms. These results indicate a role for the insula in excessive risk-avoidance relevant to OCD.

Keywords: insula, obsessive-compulsive disorder, risk processing, risk avoidance, fMRI

\section{INTRODUCTION}

Risk and the need to assess risk pervade our daily life. The outcome of choices is not always certain but different outcomes can occur probabilistically. The variance in value of these possible outcomes is a straightforward measure of the risk involved in the decision. For instance, if the choice can lead to either a very positive or a very negative outcome for the individual, there is a higher variance and therefore a higher risk than if the choice can lead to a moderately positive or a moderately negative outcome. Moreover, how the level of risk influences one's choice differs between individuals. Some may prefer low risk over high risk, for example because they overweigh the relative loss provided by the worst outcome, whereas others may be drawn to choices that involve high risk. Overall, people tend to prefer safer options, at least when the stakes are high (Pratt, 1964). This tendency toward risk avoiding strategies may have evolved because they may have promoted survival in cases where negative outcomes were life-threatening (Hintze et al., 2015). However, too much risk aversion may lead to suboptimal behavior and may be related to psychopathology. 
Clinical observations and stereotypical portraits of obsessivecompulsive disorder (OCD) in the media have led to the common-sense belief that these patients have an abnormal risk assessment: they perceive more risk, are more averse to risk, and therefore develop compulsions to prevent or avoid these perceived dangers such as contamination or harm to self or others. This believe has resonated with scientists (Steketee and Frost, 1994) and is in agreement with the finding that similar brain regions are involved in risk processing and OCD: striatum, insula, prefrontal cortex, and cingulate cortex [e.g. OCD: (Figee et al., 2011; Jung et al., 2011; Stern et al., 2011; Cocchi et al., 2012; Remijnse et al., 2013), risk processing (Preuschoff et al., 2006, 2008; d'Acremont and Bossaerts, 2008; Christopoulos et al., 2009; Tobler et al., 2009; Mohr et al., 2010; Holper et al., 2014)]. However, very little is known about the role of risk attitude and its neural correlates in OCD and available studies are inconsistent: OCD patients were either more averse to risk and showed increased amygdala activation after having made a risky choice (Admon et al., 2012) or they showed no difference in the proportion of risky choices compared to healthy controls (HCs) (Starcke et al., 2010) or difference in risk avoidance did not explain any difference in choice behavior between OCD patients and HCs (Gillan et al., 2013). Additionally, OCD is a heterogeneous disorder and it has been suggested that abnormal (i.e., heightened) risk perception may be more associated with a specific subtype of OCD characterized by worry about harm and checking compulsions (Rasmussen and Eisen, 2002). However, the relation between risk attitude in OCD and the underlying neural mechanisms of risk processing has never been investigated.

In the present study we measured risk attitude and brain activation during risk processing separately using two behavioral paradigms that exposed participants to more or less risk and functional magnetic resonance imaging (fMRI). This design enabled us to compare behavioral and neural differences in risk processing between groups and moreover to investigate whether risk attitude affected neural processing of risk differently in OCD patients compared to HCs. We hypothesize that OCD patients will be more aversive toward risk and show abnormalities in risk related brain regions during risk processing. In particular, based on the central role of the insula and the lateral prefrontal cortex in processing risk and risk attitude (Preuschoff et al., 2006; Burke and Tobler, 2011; Holper et al., 2014), we expect to find differences in risk-processing between patients and HCs in these regions.

\section{MATERIALS AND METHODS}

\section{Participants}

A total of 18 OCD patients were recruited at the Psychiatric Department of the Academic Medical Center of the University of Amsterdam and 16 control subjects were recruited from the community. Due to a hardware problem the data from the behavioral paradigm of three controls and one patient were lost. Therefore, the behavioral data and regression analysis for risk attitude and fMRI contrasts were based on 17 OCD patients and 13 control subjects. The groups were matched for age, pre-morbid intellectual functioning (IF) and gender (see Table 1). The diagnosis of OCD was established by a psychiatrist and confirmed by the Mini International Neuropsychiatric Interview (Sheehan et al., 1998; van Vliet and de Beurs, 2007) according to DSMIV criteria. Patients with a history of psychosis, bipolar disorder, developmental disorder, traumatic brain injury, or substance dependence were excluded from the study. The control group consisted of medication-free, healthy subjects without a history of OCD or any other psychiatric disorder. The study was approved by the Medical Ethics Committee of the Academic Medical Center of the University of Amsterdam and all participants gave written informed consent.

\section{Study Procedure}

On the day of testing subjects were first assessed for clinical and demographic data, then they conducted a computer task outside the scanner to assess risk attitude and finally they carried out a separate risk processing paradigm during an fMRI scanning session.

\section{Assessments \\ Clinical Characteristics}

Obsessive-compulsive disorder symptoms and OCD severity were assessed using the Yale-Brown Obsessive-Compulsive Scale and the related symptom checklist (Y-BOCS, Y-BOCS-SC (Goodman et al., 1989). The presence of anxiety and depression symptoms was assessed with the Hamilton Rating Scales for Anxiety [HAM-A (Hamilton, 1959)] and Depression [HAM-D (Hamilton, 1960)]. Pre-morbid IF was estimated using the Dutch version of the National Adult Reading Test [DART (Schmand et al., 1991)]. As expected, patients showed significantly more depression, anxiety, and obsessive-compulsive symptoms than controls (Table 1).

Ten patients were treated with serotonin reuptake inhibitors, one with a tricyclic antidepressant (clomipramine) and seven patients were unmedicated.

\section{Measuring Risk Attitude (Outside Scanner)}

Individuals differ in their choice behavior in accordance to their risk attitude: with similar expected value, risk-averse individuals prefer a low risk gamble over a high risk gamble. Risk can be mathematically defined as the variance of possible outcomes following the mean-variance approach of finance theory (Markowitz, 1959). According to this approach, we operationalized a low risk gamble by a smaller variance between two outcomes of equal probability compared to a high risk gamble. A risk-averse individual my prefer a low risk gamble, for example, because they overweigh the relative loss provided by the worst outcome whereas risk-seeking individuals prefer gambles with higher risk and may overweigh the relative gain provided by the best outcome (Christopoulos et al., 2009).

To determine risk attitude, each participant performed a computer task before the scanning session previously used by (Christopoulos et al., 2009). In each trial participants were 
TABLE 1 | Demographic and clinical data of patients and healthy controls (HCs).

\begin{tabular}{|c|c|c|c|c|c|}
\hline \multirow[t]{2}{*}{ Total group } & \multicolumn{2}{|c|}{ Patients $(N=18)$} & \multicolumn{2}{|c|}{ Controls $(N=16)$} & \multirow{2}{*}{$\begin{array}{c}\text { Difference } \\
\text { P-value }\end{array}$} \\
\hline & Mean & Range & Mean & Range & \\
\hline Age (y) & $34(6.8)$ & $23-54$ & $36(9.4)$ & $22-58$ & 0.599 \\
\hline Gender (M:F) & $6: 12$ & & $4: 12$ & & 0.595 \\
\hline Pre-morbid IF & $107(5.4)$ & $98-118$ & 109 (4.9) & $100-116$ & 0.212 \\
\hline HAM-A & 11.17 & $0-26$ & 0.44 & $0-2$ & 0.000 \\
\hline HAM-D & 9.06 & $0-24$ & 0.69 & $0-3$ & 0.000 \\
\hline YBOCS & 23.89 & 12-33 & 0 & 0 & 0.000 \\
\hline \multirow[t]{2}{*}{ Analyzed group } & \multicolumn{2}{|c|}{ Patients $(N=17)$} & \multicolumn{2}{|c|}{ Controls $(N=13)$} & Difference \\
\hline & Mean & Range & Mean & Range & $P$-value \\
\hline Age (y) & $34(7.0)$ & $23-54$ & $34(8.4)$ & $22-48$ & 0.978 \\
\hline Gender (M:F) & $6: 11$ & & $2: 11$ & & 0.222 \\
\hline Pre-morbid IF & $107(5.1)$ & $100-118$ & 108 (4.6) & $100-114$ & 0.777 \\
\hline HAM-A & 11.82 & $0-26$ & 0.38 & $0-2$ & 0.000 \\
\hline HAM-D & 9.59 & $2-24$ & 0.77 & $0-3$ & 0.000 \\
\hline YBOCS & 23.47 & $12-33$ & 0 & 0 & 0.000 \\
\hline
\end{tabular}

HAM-A, Hamilton Ratings Scale for Anxiety; HAM-D, Hamilton Ratings Scale for Depression; YBOCS, Yale-Brown Obsessive-Compulsive Scale.

presented with a gamble (two amounts with equal probability) and a safe alternative (one amount) that they had to choose from. There were three blocks with three different gambles of different risk level: 40 and 60 (low risk), 30 and 70 (medium risk), 10 and 90 (high risk), all with the same expected value of 50 and the same probability of 0.5 . In each block the gamble was kept constant while the safe amount varied in each trial according to a staircase method (parameter estimation by sequential testing: PEST) to establish the safe amount for which participants were indifferent between the gamble and safe alternative. The safe amount for which participants were indifferent between the gamble and safe amount is called the certainty equivalent (CE). For each of the three gambles (low, medium, and high risk) the CE was established. When the risk increases between the gambles a risksensitive person adapts their CE. Risk-aversion is the difference between the low risk $\mathrm{CE}$ minus the high risk $\mathrm{CE}$ and reflects how much the individual is influenced by risk. A person with no difference between the $\mathrm{CE}$ of a high and low risk gamble is unaffected by the level of risk and is therefore called risk-neutral. By contrast, a person is called risk seeking when the CE of the high risk gamble is larger than the $\mathrm{CE}$ of the low risk gamble and risk-averse when the inverse is true. For example, for the low risk gamble (40 and 60), a risk-averse person may have a CE of 45 and for the high risk gamble (10 and 90) a CE of 35, resulting in a risk-aversion of 45-35 $=10$ (Christopoulos et al., 2009).

\section{Risk Processing Paradigm (Inside Scanner)}

We adapted a paradigm used previously (Christopoulos et al., 2009; Tobler et al., 2009) which probes monetary risk processing in both choice and non-choice situations. First, participants were presented for $4.5 \mathrm{~s}$ with two gambles, both of which were made up of two monetary amounts and matched for expected value. One gamble was presented on one side and the other on the other side of the central fixation cross and participants were instructed to choose one of the two sides by button press within the $4.5 \mathrm{~s}$ (Figure 1). The response time (RT) (i.e., the time from the onset of the gambles until the button press) was measured in each trial. When participants did not respond in time they were presented with a red cross indicating their late response and the trial was repeated. Second, when participants responded in time, the gambles from the first period were presented with a red rectangular around the gamble of the chosen side for $1 \mathrm{~s}$. During the intertrial interval, which varied between 2.7 and $7.4 \mathrm{~s}$, a fixation cross was shown.

In each trial, participants had a $50 \%$ chance to win either amount on the chosen side (i.e., one of the two components of the chosen gamble). When, for example, 50 and 10 was shown on the left side and 40 and 20 on the right side, choosing the left side lead to a red rectangular around 50 and 10 on the left side and a $50 \%$ chance of winning 50 and a $50 \%$ chance of winning 10. To control for the possibility of outcome related activation contaminating risk-related activation and impacting subsequent choice behavior we did not show the outcomes of each choice. The participants were informed that at the end of the experiment, one trial would be chosen randomly and played out to determine their payoff in Euros.

In each choice trial, the presented monetary amounts made up a high or a low risk gamble, such that one side was riskier than the other. The high risk gamble was defined as $66 \%$ gain or loss relative to the expected value, whereas the low risk gamble consisted of a $33 \%$ gain or loss relative to the expected value. The gambles on each side had an expected value of either 30 (i.e., the low risk gamble had the possible outcomes of 20 and 40 whereas the high risk gamble has the possible outcomes of 10 and 50) or 60 (i.e., possible outcomes of low risk gamble: 40 and 80; possible outcomes of high risk gamble: 20 and 100). 
A

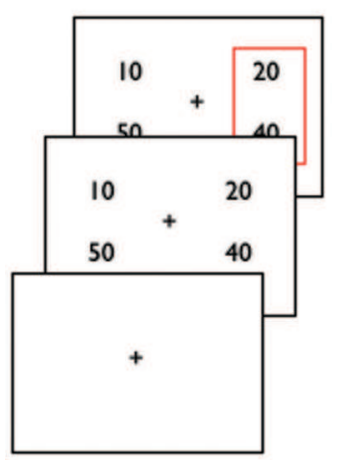

choice trial low risk
B

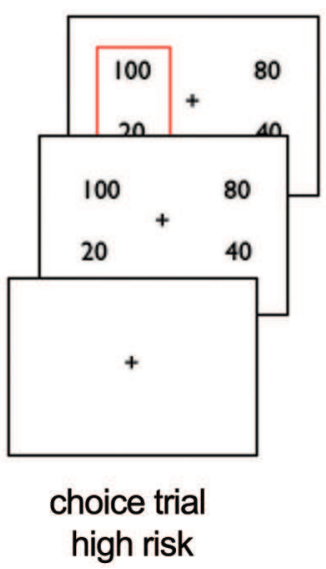

C

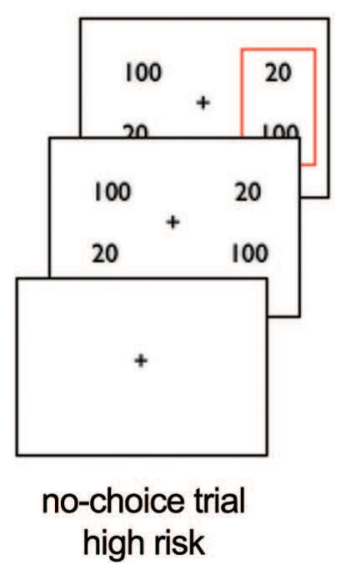

D

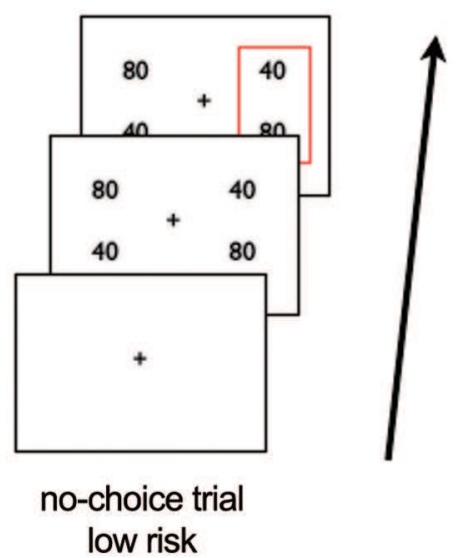

FIGURE 1 | Schematic overview of scanner task. After viewing a fixation cross participants are presented with two gambles in the first period and choose the left or right side. In choice trials $\mathbf{( A , B )}$ the gambles differ in risk, in no-choice trials (C,D) they do not. After $4.5 \mathrm{~s}$ the choice of participants is represented by a red square around the chosen gamble in slide 2. Examples of trials: (A) choice trial, participant chooses low risk gamble (B) choice trial, participant chooses high risk gamble; (C) no-choice trial, participant 'chooses' high risk gamble (D) no-choice trial, participant 'chooses' low risk gamble.

As in the original task adapted from Christopoulos et al. (2009) we included no-choice situations to expose all participants to both high and low risk. In no-choice situations the gambles on both sides were exactly the same such that participants were forced to undergo the presented risk by selecting one of the two sides (i.e., on each side 10 and 50 or 20 and 100 for high risk and 20 and 40 or 40 and 80 for low risk). In contrast, in choice situations participants could avoid exposure to specific risk levels, resulting in only high risk gamble choices (for risk seeking participants) or only low risk gamble choices (for risk-averse participants). Indeed, in the present sample, 10 participants consistently chose only high risk or only low risk gambles, which made it impossible to compare their high versus low risk trials dependent on choice. We presented two options on each side also in the no-choice trials in order to control for visual and motor factors, i.e., in both types of trials, choice and no-choice, there were two alternatives and the participants needed to select the right or left alternative. In total this resulted in 18-56 high risk trials depending on choice behavior (i.e., participants with only low risk choices would have 18 no-choice high risk trials), and 18-56 low risk trials. We used the presented level of risk as main independent variable of interest to investigate risk processing in OCD patients versus HC. The percentage of risky choices served as a proxy for risk attitude.

\section{Acquisition of Images and Pre-processing}

Magnetic Resonance Imaging data were obtained using a $3.0 \mathrm{~T}$ Intera MRI scanner (Phillips Healthcare, Best, Netherlands) equipped with a SENSE eight-channel receiver head coil. A spin echo-planar (EPI) sequence sensitive to blood oxygenation leveldependent $(\mathrm{BOLD})$ contrast $(\mathrm{TR} / \mathrm{TE}=2300 / 25 \mathrm{~ms}$, matrix size $96 \times 96$, voxel size $2.29 \mathrm{~mm} \times 2.29 \mathrm{~mm} \times 3 \mathrm{~mm}$, 40 slices, no gap) was used to acquire approximately 254 volumes and a high resolution structural scan was used for anatomical reference with EPI data.

Imaging data were analyzed using Statistical Parametric Mapping (SPM8; Wellcome Trust Centre for Neuroimaging, London, UK). Functional images of each subject were corrected for differences in slice timing, realigned, co-registered with the structural scan, segmented for normalization to an MNI template and resampled at $2 \mathrm{~mm} \times 2 \mathrm{~mm} \times 2 \mathrm{~mm}$. Finally images were smoothed using an $8 \mathrm{~mm}$ full width at half maximum Gaussian kernel.

\section{Data Analysis}

\section{Behavior}

Demographical data and behavioral performance inside and outside the scanner were analyzed using SPSS 19. Group differences in IF and age were analyzed using independent sample $t$-tests, and gender proportions were analyzed using a $\chi^{2}$ test. The significance level was set at $p<0.05$. After confirming with the Shapiro-Wilk test that the distributions of risk-aversion and CE (average CE of three risky gambles) in both groups did not significantly deviate from normality, between-group differences were analyzed with an independent sample $t$-test.

RTs in the scanning task were analyzed with a mixed model ANOVA using risk level of trial (high vs. low) as a withinparticipant variable and group as a between-participant variable. The RTs and percentages of risky choices were compared between groups with an independent sample $t$-test.

\section{Neuroimaging}

At the first level, a high-pass filter $(1 / 128 \mathrm{~Hz})$ was applied to account for low-frequency signal drift and temporal autocorrelation was modeled as an AR(1) process. The onset of the first period (i.e., presentation of gambles) was modeled with a stick function. The level of risk that participants chose (high or 
low) was our independent variable of interest and we modeled chosen risk level (high $>$ low) as a parametric modulator for both choice and no-choice trials. Risk level during presentation of gambles was therefore defined by the following behavioral choice. Specifically, we constructed a parametric modulator that assigned a 1 to all chosen high risk trials and a -1 to all chosen low risk trials. Note that in the no-choice trial participants were forced to make either a high or low risk choice by choosing from two identical options. These trials were included to expose participants to both risk levels. Therefore in our variable of interest choice and no-choice trials were used together. The six realignment parameters were included to account for head movement. Subject-specific contrasts were obtained for the parametric modulator and entered into second-level random effects analyses using an independent sample $t$-test to investigate group differences for high $>$ low risk.

In addition at the second-level a linear regression was conducted to determine whether risk attitude (i.e., level of riskaversion per subject as measured by the behavioral task, CE low - CE high) influences brain activation differently between groups in the high $>$ low risk contrast. Specifically, we used a factorial model ANOVA to assess the risk contrast, with groups (patient/controls) as between-subject factor and risk attitude as subject-specific variable.

Statistical tests were corrected for multiple comparisons across the whole brain at the cluster level $(p<0.05$, family wise error correction) using a cluster-forming threshold of $p<0.01$. The figures are presented at a threshold of $p<0.005$ uncorrected for visualization with the left side of the brain on the right side of the figures.

\section{RESULTS}

\section{Behavioral Results Outside Scanner}

The data showed that the risk attitude measures were similar for both groups: no significant difference between OCD patients and $\mathrm{HC}$ in mean risk-aversion or mean risk premium during the risk attitude assessment prior to the scanning session $(p \geq 0.25$; Table 2). In agreement with this, the ranges of risk attitudes were similar in both groups: the highest level of risk-seeking was -15 in patients and -18 in controls while the highest level of riskaversion was 20 for patients and 24 for controls. These results indicate that OCD patients are not more risk-averse than $\mathrm{HC}$ and that both groups are heterogeneous and vary considerably in their risk attitudes.

\section{Behavioral Results Inside Scanner}

In line with similar risk attitudes in both groups prior to scanning, we also observed no significant group differences in the percentage of high risk choices during the task in the scanner (Table 2). Additionally we found a positive correlation at trend significance $(r=0.292 p=0.124)$ for risk aversion (measured outside scanner) and percentage of safe choices made in choice trials (measured inside scanner).

RTs differed between high risk versus low risk gambles with shorter RTs for selecting the high risk gamble. This indicates that participants respond differently to high risk compared to low risk validating the risk manipulation. However, there were no significant group differences or group $\times$ condition interaction effects.

\section{Neural Correlates of Risk Processing}

We examined neural processing of risk while participants were exposed to high versus low risk (Figure 1). No significant main effects and no significant differences between patients and controls were found when we compared brain activation induced by high versus low risk. Thus, we found no indications for neural differences in risk processing between the groups.

\section{Effect of Risk Attitude on Risk Processing}

To test whether risk attitude affected neural processing of risk differently across groups we performed a linear regression analysis between risk attitude (i.e., level of risk-aversion) and the high versus low risk fMRI contrast. This revealed an interaction effect with group: patients showed a stronger correlation between risk-aversion and brain activation (high risk $>$ low risk) than controls in the insula $\left(T=5.95, P_{\mathrm{FWE}}<0.001\right)$, dorsolateral prefrontal cortex $\left(T=5.99, P_{\mathrm{FWE}}<0.001\right)$ and preand postcentral gyrus $\left(T=4.33, P_{\mathrm{FWE}}=0.001 ; T=4.23\right.$, $P_{\text {FWE }}<0.032$ : Figures $2 \mathrm{~A}$ and 3; Table 3; all statistical tests were whole-brain cluster-level corrected). These results remained significant after controlling for differences in anxiety and depression scores. No significantly stronger correlations were found for the controls compared to patients.

In order to determine the direction of the association within groups we performed follow-up testing which showed a positive correlation between insula activity during risk processing and risk-aversion in patients $\left(T=6.11, P_{\mathrm{FWE}}=0.001\right.$ : Figures $2 \mathrm{~B}$ and 3A; Table 3), and a negative correlation between risk-aversion and activation of this region for controls $\left(T=5.64, P_{\mathrm{FWE}}=0.047\right.$ : Figures 2B and 3B; Table 3). Patients also showed a positive correlation between risk-aversion and activity in the DLPFC $\left(T=5.57, P_{\mathrm{FWE}}=0.004\right)$ and activity in the precentral gyrus $\left(T=4.34, P_{\mathrm{FWE}}=0.021\right.$ : Table 3; Figure 2C).

These results imply that patients and controls show an opposite pattern of insula recruitment during risk processing: high risk situations resulted in higher insula activation in riskaverse patients while high risk situations resulted in lower insula activation in risk-averse HC. Moreover compared to HC, patients showed stronger activation increases in the DLPFC and pre/postcentral gyrus with risk-aversion during risk processing (Table 3 and Figures 2A,C).

\section{Post Hoc Exploration of Risk Attitude and Clinical Data in the Patient Group}

The lack of differences in risk attitude between groups may be due to the heterogeneity of the disorder where different subgroups of OCD show different levels of risk attitude. To explore this hypothesis, patients were allocated to one of five OCD symptom dimensions: (i) hoarding, (ii) contamination/cleaning, (iii) symmetry/ordering, (iv) 
TABLE 2 | Risk attitude and behavioral results of risk processing paradigm.

\begin{tabular}{|c|c|c|c|c|c|}
\hline \multirow[t]{2}{*}{ Risk attitude (outside scanner) } & \multicolumn{2}{|c|}{ Patients $(N=17)$} & \multicolumn{2}{|c|}{ Controls $(N=13)$} & \multirow{2}{*}{$\begin{array}{c}\text { Difference } \\
\text { P-value }\end{array}$} \\
\hline & Mean (SD) & Range & Mean (SD) & Range & \\
\hline Risk aversion (high-low premium) & $-0.59(9.4)$ & -15 to 20 & $3.46(12.6)$ & -18 to 24 & 0.32 \\
\hline \multirow[t]{2}{*}{ Risk processing paradigm (inside scanner) } & \multicolumn{2}{|c|}{ Patients $(N=18)$} & \multicolumn{2}{|c|}{ Controls $(N=16)$} & Difference \\
\hline & Mean (SD) & Range & Mean (SD) & Range & $P$-value \\
\hline Risky choices (\%) & $35.73(32.45)$ & $0-100$ & $22.36(28.89)$ & $0-100$ & 0.21 \\
\hline RT Risk * Group & & & & & 0.68 \\
\hline RT Risk & & & & & $0.04^{1}$ \\
\hline Group: RT high risk (s) & $1.625(0.23)$ & $1.26-2.09$ & $1.54(0.24)$ & $1.16-1.96$ & 0.29 \\
\hline Group: RT low risk (s) & $1.690(0.29)$ & $1.33-2.41$ & $1.58(0.30)$ & $1.11-2.15$ & 0.29 \\
\hline
\end{tabular}

$R T$, reaction time; ${ }^{1}$ significant $(p<0.05)$.

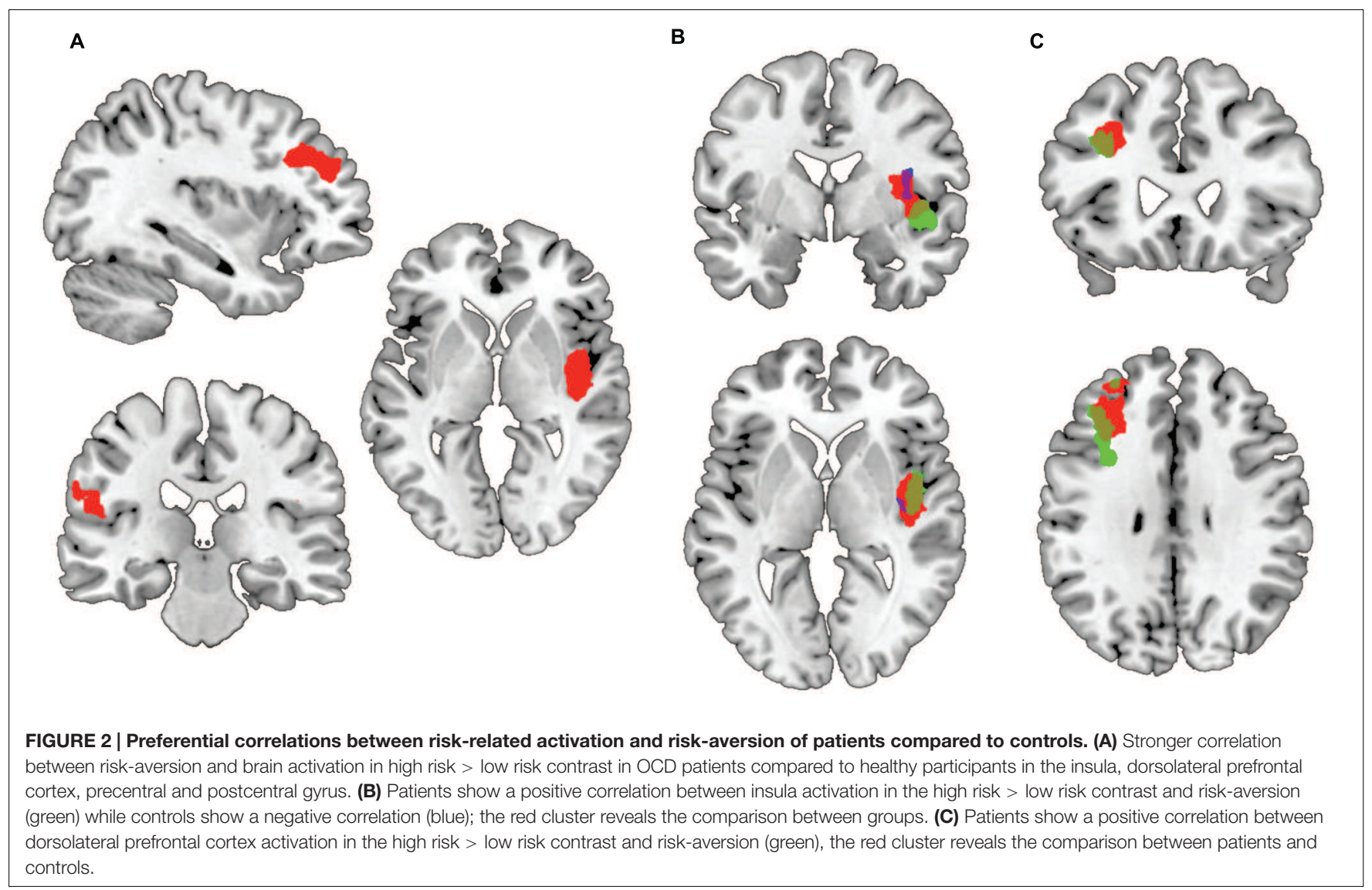

unacceptable/taboo thoughts (v) doubt/checking according to the YBOCS symptoms checklist (Brakoulias et al., 2013). When patients scored on multiple dimensions, they were assigned to the dimension for which they reported most symptoms. We excluded patients with predominantly hoarding symptoms, as there is evidence that this dimension may be independent from OCD (Pertusa et al., 2008). All seven risk-averse OCD patients belonged to the doubt/checking subgroup, whereas the 10 patients in the risk-seeking group consisted of three patients with mainly unacceptable/taboo thoughts, two with mainly symmetry/ordering symptoms, three with mainly contamination/cleaning symptoms, and only two with mainly doubt/checking symptoms (Supplementary Table S1). Note that the two risk-seeking patients with mainly doubt/checking symptoms were close to being risk-neutral. Accordingly, risk-averse and risk-seeking patients showed a significant difference in symptom dimension $(p=0.014)$. On average, patients with doubt/checking symptoms were significantly more risk-averse than patients with other symptoms $(p<0.001)$, and this remained significant after controlling for whether patients 


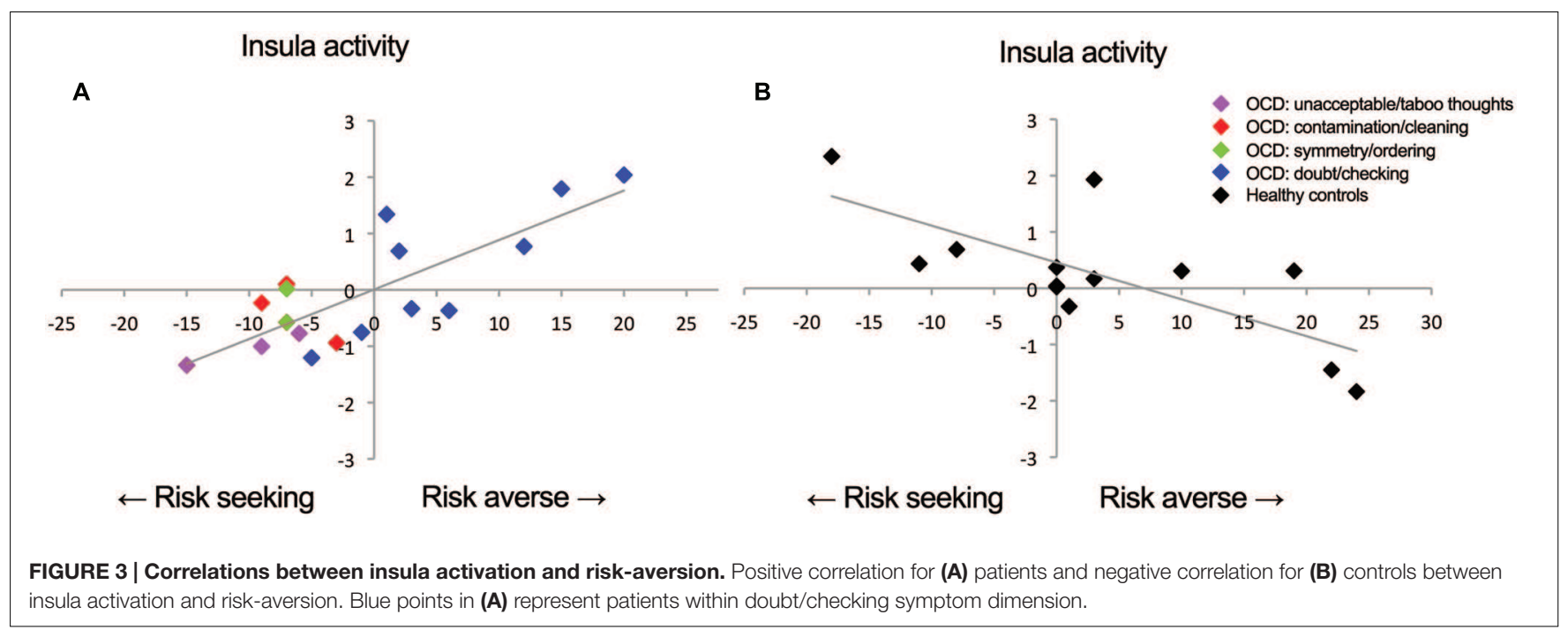

TABLE 3 | Comparison of regression analysis between groups.

\begin{tabular}{|c|c|c|c|c|c|c|c|c|c|}
\hline Test & Direction of correlation & Region & Side & Cluster level $P$ value (FWE) & $T$ & Cluster size & \multicolumn{3}{|c|}{ MNI } \\
\hline & \multirow{3}{*}{ Patients $>$ controls } & DLPFC & $\mathrm{L}$ & 0.000 & 5.99 & 840 & -32 & 28 & 32 \\
\hline & & Precentral gyrus & $L$ & 0.001 & 4.33 & 682 & -64 & -30 & 26 \\
\hline & & Pre/postcentral gyrus & $\mathrm{L}$ & 0.032 & 4.23 & 407 & -48 & -4 & 44 \\
\hline & \multirow{2}{*}{ Positive } & DLPFC & $\mathrm{L}$ & 0.004 & 5.57 & 579 & -34 & 26 & 30 \\
\hline & & Precentral gyrus & $L$ & 0.021 & 4.34 & 433 & -64 & -28 & 30 \\
\hline
\end{tabular}

Comparison of regression analysis of brain activation in the high-risk > low-risk contrast against risk aversion between groups. R, right; L, left; DLPFC, dorsolateral prefrontal cortex. For overlap between different insula and DLPFC clusters see Figures 2B,C.

used medication ( $p=0.001$ ), indicating that the differences between groups were not due to differences in medication use.

To test whether medication use affected risk attitude or the number of risky choices in the scanner we used an independent sample $t$-test to compare medicated and unmedicated OCD patients. We found no differences in mean risk-aversion or percentage of risky choices in the scanner between medicated and unmedicated OCD patients: -1.3 (10.4) vs. $0.7(7.9)(p=0.70)$; risky choices, medicated and unmedicated patients: $39.4 \%$ (37.9) vs. $28.3 \%(18.1)(p=0.41)$.

\section{DISCUSSION}

Contrary to common belief, patients with OCD were not more averse to risk than $\mathrm{HC}$ in the present task. Regardless, $\mathrm{HC}$ and OCD patients showed an opposite correlation between riskaversion and insula activity during risk processing: insula activity correlated positively with risk-aversion in patients, whereas in $\mathrm{HC}$ insula activity correlated negatively with risk-aversion. Moreover OCD patients showed stronger activation increases in the DLPFC and pre/postcentral gyrus with risk-aversion during risk processing.

Patients showed stronger activation in the right insula to high versus low risk with increasing risk-aversion, whereas controls showed stronger activation in the same region with increasing propensity to seek risk (i.e., decreasing risk-aversion). Growing evidence suggests that the insula is involved in interoceptive processing (i.e., perception of internal feelings of the body) and the evaluation of interoceptive states contributing to subjective feelings and emotions (Craig, 2002). Additionally neuroeconomic studies have pointed to a role of the insula in risk processing (Kuhnen and Knutson, 2005; Huettel, 2006; Preuschoff et al., 2008; Burke and Tobler, 2011). Risk processing in humans is not only a deliberate calculation of probability but also involves the evaluation of affective states (Mukherjee, 2010). Taken together these findings have led to the hypothesis that the insula is critically involved in the affective processes underlying risk processing (Paulus et al., 2003; Gowin et al., 2014). Additionally the insula has been related to cognitive control and the accumulation of information in the process of decision making possibly affecting risk taking (Hu et al., 2014). Our data concur and raise the possibility 
that interoceptive processes may play a particularly prominent role in the subgroup of OCD patients with doubt and checking symptoms.

Moreover, the insula may also be involved in expressing the affective components of risk into behavior. In agreement with this notion, evidence suggests that activity in the insula may be associated with an urge for risk taking in $\mathrm{HC}$ (Xue et al., 2010) and in non-human species (Ishii et al., 2012) which is in line with our finding that risk-related insula activation is associated with risk-seeking in HC. The finding that activity in the same region of the insula in patients is correlated with risk-aversion may suggest that at least some parts of the insula assume a differential role in the two groups: for $\mathrm{HC}$ this subregion may bias behavior towards taking risks whereas for OCD patients it could bias behavior towards risk avoidance. Alternatively, in both groups the insula may signal general arousal or decision urgency but this is experienced more strongly by risk-averse patients and by risk-seeking HC participants. In both cases, the insula seems to play an important role in the integration of bodily interoceptive signals with awareness appropriate action tendencies (i.e., approach or avoid) in the face of high risk. The insula may play such a role (Xue et al., 2010; Ishii et al., 2012) in an individually adjusted manner (Paulus et al., 2003; Kuhnen and Knutson, 2005).

Additionally, risk-averse OCD patients showed increased recruitment of the DLPFC and precentral gyrus during risk processing in contrast to HCs. This contrasts with HCs (Tobler et al., 2009; Holper et al., 2014) and may reflect increased collaboration between prefrontal regions and the insula when patients process risk. The DLPFC has previously been shown to encode the value of risk (Huettel, 2006; Christopoulos et al., 2009; Tobler et al., 2009; Holper et al., 2014). Moreover DLPFC has been implicated in executive functioning and specifically cognitive control (Mohr et al., 2010). Speculatively, the increased recruitment of this region in risk-averse OCD patients might show additional control mechanisms in the face of risky choices.

No differences in risk attitude were found between groups, suggesting that in general, OCD patients are not more risk averse than HCs. Consistent with the behavioral results no overall neural differences were found during risk processing between groups. A possible explanation for this lack of differences between groups but the finding that the groups differ in correlation between risk attitude and neural correlates is that OCD is a heterogeneous disorder and different subtypes of OCD show differences in risk attitude. Indeed post hoc analyses showed that all risk-averse OCD patients expressed doubt/checking symptoms and this subgroup was more riskaverse than patients with other symptoms. Patients with doubt/checking symptoms report obsessions about causing unintentional harm to others, fear that something terrible might happen, indecisiveness and checking compulsions. Interestingly, the heightened risk-aversion of this group became apparent here even in situations in which only gains could occur. The finding that risk-aversion may contribute to only a specific subtype of OCD suggests that for this group addressing abnormal risk-assessment in cognitive behavioral therapy may be helpful.

Several limitations are worth mentioning. One potential limitation of our study was the relatively strong behavioral consistency of our subjects, which made it impossible to investigate potential interactions between risk and choice. Additionally, in the paradigm used in this study, risk arose from the variance of money that could be earned while there was no risk of losing money. Therefore risk-aversion was not based on loss prevention but on a preference for more certainty in gain. In a pure gain context, risk-aversion could result from perceiving the lowest possible outcome as relative loss or from perceiving more variance. OCD patients may have different neural responses during actual loss versus reward anticipation (Choi et al., 2012) and including losses could have affected risky choices in OCD irrespective of symptoms. However, a previous study (Gillan et al., 2013) using both gains and losses nevertheless confirmed our result that on average risk processing is unaffected in OCD. A further limitation could be that the range between risk-seeking and risk-averse extremes was higher in both the OCD and HC group than expected based on a previous study using a similar task (Christopoulos et al., 2009). This increased variance in risk attitude may be due to the heterogeneity of our group in terms of age and IF compared to the group of primarily college students used in the previous study.

Another potential limitation is the fact that 10 patients were using serotonin-reuptake inhibitors (SRIs) and one patient was using a tricyclic antidepressant whereas 7 other patients did not receive medication. Serotonin neurotransmission is correlated with successful withholding of responses and risk avoidance, whereas low serotonin promotes early responding and risk taking (Cools et al., 2008; Long et al., 2009). In the present study we did not find any differences in riskaversion or propensity for risky choice between medicated and unmedicated patients and differences in risk-aversion between patients with doubt/checking symptoms and other patients remained significant after controlling for medication use. Therefore it seems unlikely that in our study SRI medication explains the risk profiles of patients. However, for comparisons within the OCD group the sample size is small and this has to be taken into account when interpreting the differences between risk-averse and risk-seeking patients.

In conclusion, we found elevated insula activation during risk processing in risk-averse OCD patients, which may suggest that the insula is involved in an increased urge to avoid risk in these patients. Increased avoidance signaling in the insula might contribute to the development of risk-avoidant strategies in this group, which in turn could lead to persistence of the disorder.

\section{AUTHOR CONTRIBUTIONS}

JL analyzed the data and wrote the manuscript. MF initiated the study and revised the manuscript. PT designed the experiment 
and revised the paper. GW drafted and revised the paper. WB revised the paper. BK collected the data. DD initiated the study and revised the paper.

\section{FUNDING}

Research in this paper was supported in part by grant 916.66 .095 from the Netherlands Organization for Scientific Research ZONMW VENI program and by grant PP00P1_128574 from the Swiss National Science Foundation.

\section{REFERENCES}

Admon, R., Bleich-Cohen, M., Weizmant, R., Poyurovsky, M., Faragian, S., and Hendler, T. (2012). Functional and structural neural indices of risk aversion in obsessive-compulsive disorder (OCD). Psychiatry Res. 203, 207-213. doi: 10.1016/j.pscychresns.2012.02.002

Brakoulias, V., Starcevic, V., Berle, D., Sammut, P., Milicevic, D., Moses, K., et al. (2013). Further support for five dimensions of obsessive-compulsive symptoms. J. Nerv. Ment. Dis. 201, 452-459. doi: 10.1097/NMD.0b013e318294804e

Burke, C. J., and Tobler, P. N. (2011). Reward skewness coding in the insula independent of probability and loss. J. Neurophysiol. 106, 2415-2422. doi: 10.1152/jn.00471.2011

Choi, J.-S., Shin, Y.-C., Jung, W. H., Jang, J. H., Kang, D.-H., Choi, C.H., et al. (2012). Altered brain activity during reward anticipation in pathological gambling and obsessive-compulsive disorder. PLoS One 7:e45938. doi: 10.1371/journal.pone.0045938

Christopoulos, G. I., Tobler, P. N., Bossaerts, P., Dolan, R. J., and Schultz, W. (2009). Neural correlates of value, risk, and risk aversion contributing to decision making under risk. J. Neurosci. 29, 12574-12583. doi: 10.1523/JNEUROSCI.2614-09

Cocchi, L., Harrison, B. J., Pujol, J., Harding, I. H., Fornito, A., Pantelis, C., et al. (2012). Functional alterations of large-scale brain networks related to cognitive control in obsessive-compulsive disorder. Hum. Brain Mapp. 33, 1089-1106. doi: $10.1002 / \mathrm{hbm} .21270$

Cools, R., Roberts, A. C., and Robbins, T. W. (2008). Serotoninergic regulation of emotional and behavioural control processes. Trends Cogn. Sci. 12, 31-40. doi: 10.1016/j.tics.2007.10.011

Craig, A. D. (2002). How do you feel? Interoception: the sense of the physiological condition of the body. Nat. Rev. Neurosci. 3, 655-666. doi: 10.1038/nrn894

d'Acremont, M., and Bossaerts, P. (2008). Neurobiological studies of risk assessment: a comparison of expected utility and mean-variance approaches. Cogn. Affect. Behav. Neurosci. 8, 363-374. doi: 10.3758/CABN.8.4.363

Figee, M., Vink, M., de Geus, F., Vulink, N., Veltman, D. J., Westenberg, H., et al. (2011). Dysfunctional reward circuitry in obsessive-compulsive disorder. Biol. Psychiatry 69, 867-874. doi: 10.1016/j.biopsych.2010.12.003

Gillan, C. M., Morein-Zamir, S., Kaser, M., Fineberg, N. A., Sule, A., Sahakian, B. J., et al. (2013). Counterfactual processing of economic actionoutcome alternatives in obsessive-compulsive disorder: further evidence of impaired goal-directed behavior. Biol. Psychiatry 75, 639-646. doi: 10.1016/j.biopsych.2013.01.018

Goodman, W. K, Price, L. H., Rasmussen, S. A., Mazure, C., Delgado, P., Heninger, G. R., et al. (1989). The yale-brown obsessive compulsive scale: II. Validity. Arch. Gen. Psychiatry 46, 1012-1016. doi: 10.1001/archpsyc.1989.018101100 54008

Gowin, J. L., Harlé, K. M., Stewart, J. L., Wittmann, M., Tapert, S. F., and Paulus, M. P. (2014). Attenuated insular processing during risk predicts relapse in early abstinent methamphetamine dependent individuals. Neuropsychopharmacology 39. 1379-1387. doi: 10.1038/npp.2013.333

Hamilton, M. (1959). The assessment of anxiety states by rating. Br. J. Med. Psychol. 32, 50-55. doi: 10.1111/j.2044-8341.1959.tb00467.x

Hamilton, M. (1960). A rating scale for depression. J. Neurol. Neurosurg. Psychiatry 23, 56-62. doi: 10.1136/jnnp.23.1.56

\section{ACKNOWLEDGMENT}

We thank Milenna van Dijk for her assistance with data collection.

\section{SUPPLEMENTARY MATERIAL}

The Supplementary Material for this article can be found online at: http://journal.frontiersin.org/article/10.3389/fnhum. 2016.00283

Hintze, A., Olson, R. S., Adami, C., and Hertwig, R. (2015). Risk sensitivity as an evolutionary adaptation. Sci. Rep. 5, 8242. doi: 10.1038/srep 08242

Holper, L., Wolf, M., and Tobler, P. N. (2014). Comparison of functional nearinfrared spectroscopy and electrodermal activity in assessing objective versus subjective risk during risky financial decisions. Neuroimage 84, 833-842. doi: 10.1016/j.neuroimage.2013.09.047

Hu, S., Tseng, Y.-C., Winkler, A. D., and Li, C.-S. R. (2014). Neural bases of individual variation in decision time. Hum. Brain Mapp. 35, 2531-2542. doi: 10.1002/hbm. 22347

Huettel, S. A. (2006). Behavioral, but not reward, risk modulates activation of prefrontal, parietal, and insular cortices. Cogn. Affect. Behav. Neurosci 6, $141-$ 151. doi: 10.3758/CABN.6.2.141

Ishii, H., Ohara, S., Tobler, P. N., Tsutsui, K.-I., and Iijima, T. (2012). Inactivating anterior insular cortex reduces risk taking. J. Neurosci. 32, 16031-16039. doi: 10.1523/JNEUROSCI.2278-12.2012

Jung, W. H., Kang, D.-H., Han, J. Y., Jang, J. H., Gu, B.-M., Choi, J.-S., et al. (2011). Aberrant ventral striatal responses during incentive processing in unmedicated patients with obsessive-compulsive disorder. Acta Psychiatr. Scand. 123, 376386. doi: 10.1111/j.1600-0447.2010.01659.x

Kuhnen, C. M., and Knutson, B. (2005). The neural basis of financial risk taking. Neuron 47, 763-770. doi: 10.1016/j.neuron.2005.08.008

Long, A. B., Kuhn, C. M., and Platt, M. L. (2009). Serotonin shapes risky decision making in monkeys. Soc. Cogn. Affect. Neurosci. 4, 346-356. doi: 10.1093/scan/nsp020

Markowitz, H. (1959). Portfolio Selection: Efficient Diversification of Investments. Available at: http://www.citeulike.org/group/2384/article/1204370 [accessed June 24, 2014].

Mohr, P. N. C., Biele, G., and Heekeren, H. R. (2010). Neural processing of risk. J. Neurosci. 30, 6613-6619. doi: 10.1523/JNEUROSCI.0003-10. 2010

Mukherjee, K. (2010). A dual system model of preferences under risk. Psychol. Rev. 117, 243-255. doi: 10.1037/a0017884

Paulus, M. P., Rogalsky, C., Simmons, A., Feinstein, J. S., and Stein, M. B. (2003). Increased activation in the right insula during risk-taking decision making is related to harm avoidance and neuroticism. Neuroimage 19, 1439-1448. doi: 10.1016/S1053-8119(03)00251-9

Pertusa, A., Fullana, M. A., Singh, S., Alonso, P., Menchón, J. M., and Mataix-Cols, D. (2008). Compulsive hoarding: OCD symptom, distinct clinical syndrome, or both? Am. J. Psychiatry 165, 1289-1298. doi: 10.1176/appi.ajp.2008.07111730

Pratt, J. W. (1964). Risk aversion in the small and in the large. Econometrica 32, 122-136.

Preuschoff, K., Bossaerts, P., and Quartz, S. R. (2006). Neural differentiation of expected reward and risk in human subcortical structures. Neuron 51, 381-390. doi: 10.1016/j.neuron.2006.06.024

Preuschoff, K., Quartz, S. R., and Bossaerts, P. (2008). Human insula activation reflects risk prediction errors as well as risk. J. Neurosci. 28, 2745-2752. doi: 10.1523/JNEUROSCI.4286-07.2008

Rasmussen, S., and Eisen, J. (2002). "The course and clinical features of obsessivecompulsive disorder," in Neuropsychopharmacology - 5th Generation of Progress (Philadelphia, PA: Lippincott, Williams \& Wilkins), 1593-1607. 
Remijnse, P. L., van den Heuvel, O. A., Nielen, M. M. A., Vriend, C., Hendriks, G.-J., Hoogendijk, W. J. G., et al. (2013). Cognitive inflexibility in obsessivecompulsive disorder and major depression is associated with distinct neural correlates. PLoS ONE 8:e59600. doi: 10.1371/journal.pone.0059600

Schmand, B., Bakker, D., Saan, R., and Louman, J. (1991). [The Dutch Reading Test for Adults: a measure of premorbid intelligence level]. Tijdschr. Gerontol. Geriatr. 22, 15-19.

Sheehan, D. V., Lecrubier, Y., Sheehan, K. H., Amorim, P., Janavs, J., Weiller, E., et al. (1998). The Mini-International Neuropsychiatric Interview (M.I.N.I.): the development and validation of a structured diagnostic psychiatric interview for dsm-iv and icd-10. J. Clin. Psychiatry 59(Suppl. 20), 22-33; quiz 34-57.

Starcke, K., Tuschen-Caffier, B., Markowitsch, H. J., and Brand, M. (2010). Dissociation of decisions in ambiguous and risky situations in obsessive-compulsive disorder. Psychiatry Res. 175, 114-120. doi: 10.1016/j.psychres.2008.10.022

Steketee, G., and Frost, R. O. (1994). Measurement of risk-taking in obsessive-compulsive disorder. Behav. Cogn. Psychother. 22, 287-298. doi: $10.1017 /$ S1352465800013175

Stern, E. R., Welsh, R. C., Fitzgerald, K. D., Gehring, W. J., Lister, J. J., Himle, J. A., et al. (2011). Hyperactive error responses and altered connectivity in ventromedial and frontoinsular cortices in obsessive-compulsive disorder. Biol. Psychiatry 69, 583-591. doi: 10.1016/j.biopsych.2010.09.048
Tobler, P. N., Christopoulos, G. I., O'Doherty, J. P., Dolan, R. J., and Schultz, W. (2009). Risk-dependent reward value signal in human prefrontal cortex. Proc. Natl. Acad. Sci. U.S.A. 106, 7185-7190. doi: 10.1073/pnas.08095 99106

van Vliet, I. M., and de Beurs, E. (2007). [The MINI-international neuropsychiatric interview. a brief structured diagnostic psychiatric interview for DSM-IV en ICD-10 psychiatric disorders]. Tijdschr. Psychiatr. 49, 393-397.

Xue, G., Lu, Z., Levin, I. P., and Bechara, A. (2010). The impact of prior risk experiences on subsequent risky decision-making: the role of the Insula. Neuroimage 50, 709-716. doi: 10.1016/j.neuroimage.2009.12.097

Conflict of Interest Statement: The authors declare that the research was conducted in the absence of any commercial or financial relationships that could be construed as a potential conflict of interest.

Copyright $\odot 2016$ Luigjes, Figee, Tobler, van den Brink, de Kwaasteniet, van Wingen and Denys. This is an open-access article distributed under the terms of the Creative Commons Attribution License (CC BY). The use, distribution or reproduction in other forums is permitted, provided the original author(s) or licensor are credited and that the original publication in this journal is cited, in accordance with accepted academic practice. No use, distribution or reproduction is permitted which does not comply with these terms. 